\title{
Erratum to: $\beta$-Amyloid triggers aberrant over-scaling of homeostatic synaptic plasticity
}

\author{
James Gilbert ${ }^{1}$, Shu Shu ${ }^{2}$, Xin Yang ${ }^{2}$, Youming Lu, ${ }^{2,5}$, Ling-Qiang Zhu ${ }^{3,5}$ and Heng-Ye Man ${ }^{1,4,5^{*}}$
}

\section{Erratum}

In the original publication of this article [1], Fig. 4b contains a recording trace which was duplicated. In this Erratum the original Fig. 4b (Fig. 1) and the corrected Fig. 4b (Fig. 2) are published.

\footnotetext{
Author details

'Department of Biology, Boston University, Boston, MA, USA. ${ }^{2}$ Department of Physiology, School of Basic Medicine, Tongji Medical College, Huazhong University of Science and Technology, Wuhan 430030, People's Republic of China. ${ }^{3}$ Department of Pathophysiology, School of Basic Medicine, Tongji Medical College, Huazhong University of Science and Technology, Wuhan 430030, People's Republic of China. ${ }^{4}$ Department of Pharmacology \& Experimental Therapeutics, Boston University School of Medicine, Boston, MA, USA. ${ }^{5}$ The Institute for Brain Research, Collaborative Innovation Center for Brain Science, Huazhong University of Science and Technology, Wuhan 430030, People's Republic of China.
}

Published online: 09 March 2017

\section{Reference}

1. Gillbert et al. (2016) $\beta$-Amyloid triggers aberrant over-scaling of homeostatic synaptic plasticity. Acta Neuropathol Commun 4:131. doi: 10.1186/s40478-016-0398-0

\footnotetext{
*Correspondence: hman@bu.edu

'Department of Biology, Boston University, Boston, MA, USA

${ }^{4}$ Department of Pharmacology \& Experimental Therapeutics, Boston

University School of Medicine, Boston, MA, USA
} 


\section{A1 $24 \mathrm{~h} \mathrm{VD+Vehicle} \mathrm{or} \mathrm{VD+A \beta}$}

A2

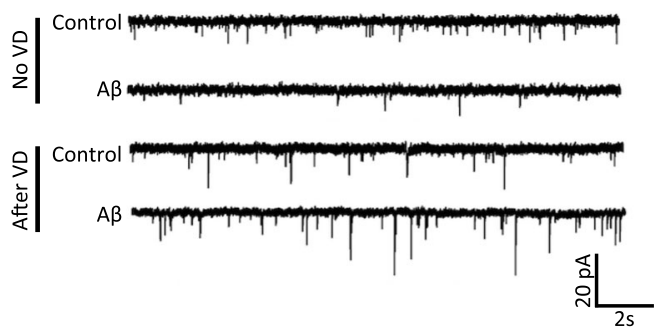

B1

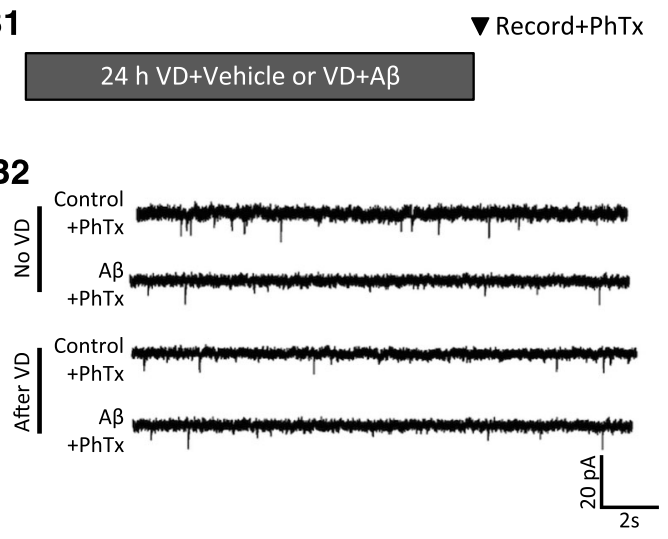

A3
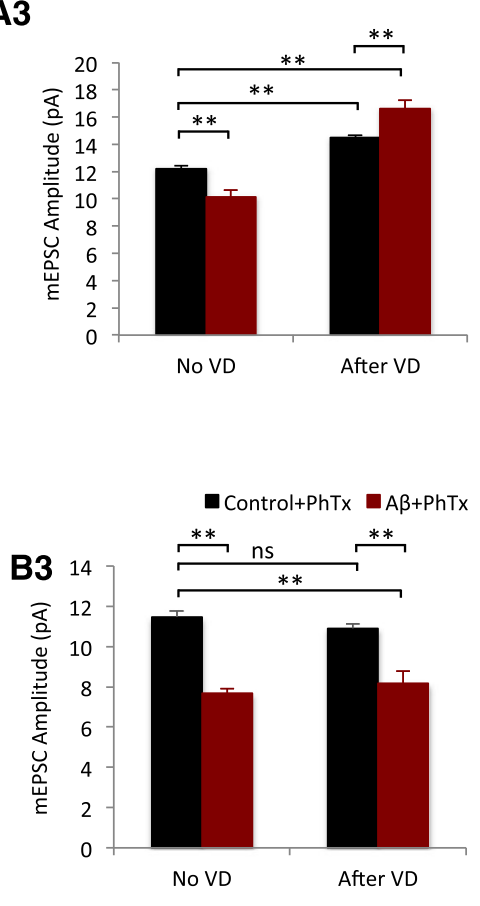

Fig. 1 Original version of Fig. 4b as published on 13 December 2016 


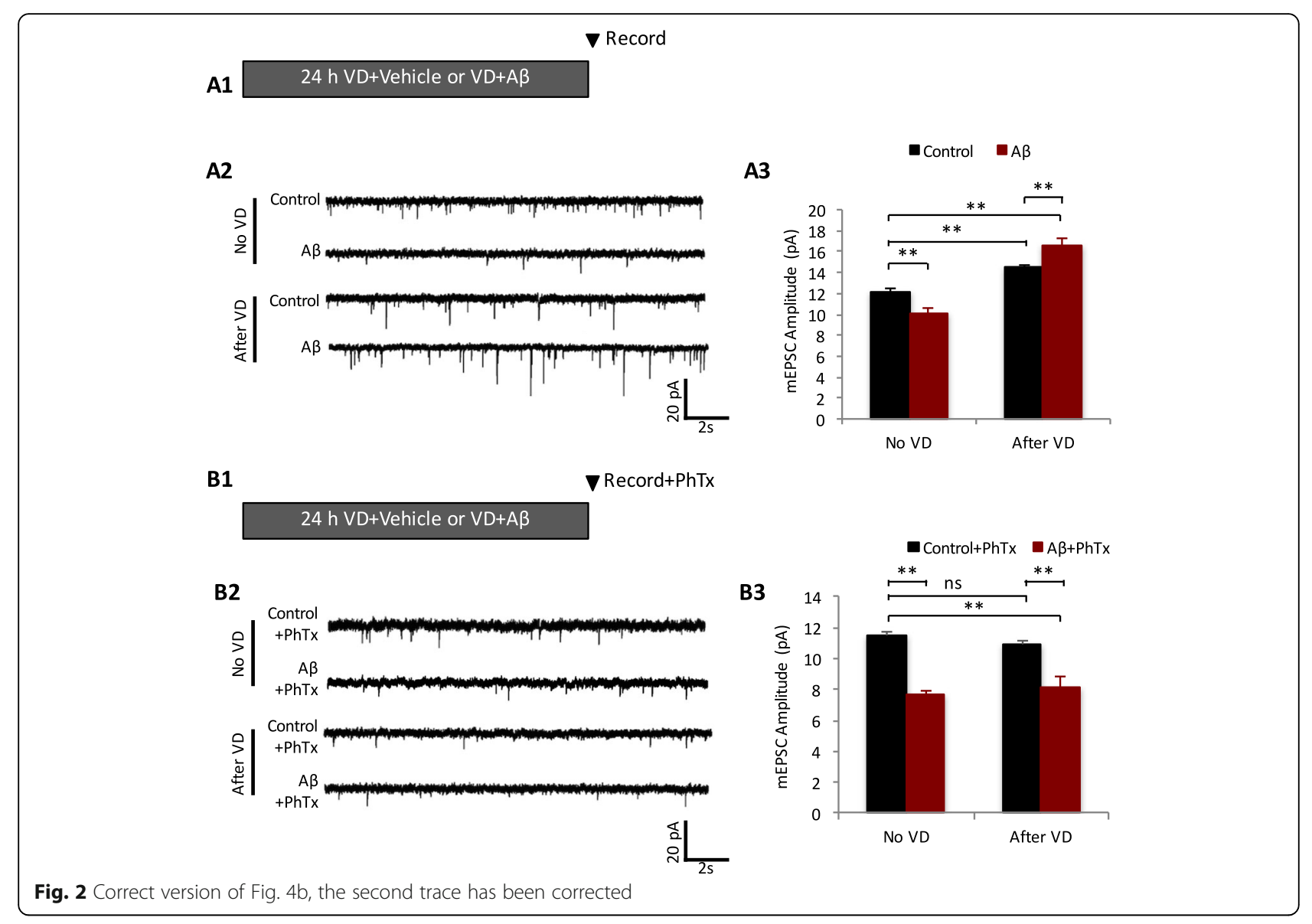

